\section{Variability of disease activity in patients treated with ranibizumab for neovascular age-related macular degeneration}

Department of

Ophthalmology, University

Hospital of Cologne,

Cologne, Germany

Correspondence:

S Fauser, Department of Ophthalmology, University Hospital of Cologne,

Kerpener Street 62, Cologne 50924, Germany

Tel: +49 2214784105 ;

Fax: +49221478 5922

E-mail: sascha.fauser@

gmail.com

Received: 15 August 2015 Accepted in revised form: 25 February 2016

Published online:

20 May 2016

\begin{abstract}
Purpose To analyze choroidal neovasularization (CNV) activity and recurrence patterns in patients with neovascular age-related macular degeneration (nAMD) treated with ranibizumab, and the correlation with individual intraocular vascular endothelial growth factor (VEGF) suppression time (VST).

Methods Post-hoc analysis of data from a prospective, non-randomized clinical study. Patients with nAMD treated with ranibizumab on a pro re nata regimen. Disease activity was analyzed monthly by spectral-domain optical coherence tomography and correlated with VSTs. Results Overall, 73 eyes of 73 patients were included in the study with a mean follow-up of 717 days (range: 412-1239 days). Overall, the mean $\mathrm{CNV}$-activity-free interval was 76.5 days (range: 0-829 days). The individual range of the length of dry intervals was high. A total of $42 \%$ of patients had a range of more than $\mathbf{9 0}$ days. Overall, $16 \%$ of patients showed persistent activity. And $12 \%$ stayed dry after the initial ranibizumab treatment. No significant correlation was found between the CNVrecurrence pattern and VST $(P=0.12)$. Conclusions CNV activity in nAMD is irregular, which is reflected in the range of the duration of dry intervals and late recurrences. The biomarker VST solely seems not to be sufficient to explain recurrence pattern of $\mathrm{CNV}$ in all AMD patients.
\end{abstract}

Eye (2016) 30, 1072-1076; doi:10.1038/eye.2016.97; published online 20 May 2016
P Enders, P Scholz, PS Muether and S Fauser

\section{Introduction}

Neovascular age-related macular degeneration (nAMD) is a common cause of vision loss. The disease is treated with drugs targeting vascular endothelial growth factor (VEGF) such as ranibizumab. Although initially the drug was injected monthly in a fixed scheme, there are now several regimens monitoring disease activity and dosing the drug as needed (pro re nata). ${ }^{1}$ Most regimens require monthly visits for either treatment or monitoring in order not to miss recurrent disease activity because a delay of therapy can result in permanent vision loss. ${ }^{2,3}$ In clinical trials these monthly visits can be achieved but in real life patients are often treated and monitored less frequently. ${ }^{4,5}$ Therefore, alternative regimens would be preferable reducing the burden for the patient. In the 'treat and extend' regimen, injections are given until an inactive disease is achieved. Treatment intervals are then gradually extended until recurrent activity is detected, then the treatment interval is shortened again. Overall, this approach can reduce the number of visits. This regimen looks for patient-specific dry intervals, which ideally would be regular and stable over a long period of time. ${ }^{6-8}$

It would facilitate therapy if need for treatment could be predicted for any individual patient. Local or systemic biomarkers are currently in scope of research to improve predictability of choroidal neovasularization $(\mathrm{CNV})$ recurrence. So far there is little data on predictors for the individual response to antiVEGF treatment. However, we have recently shown that ranibizumab suppresses VEGF in the 
aqueous humor of the eye for 37 days on average (VEGF suppression times, VSTs) with individual VSTs ranging from 26 to 69 days. VSTs in individual patients show high levels of stability over a period of up to three years. ${ }^{9,10}$

If VSTs would correlate with recurrent CNV activity, they might be used to specify individualized treatment intervals.

In this study, we analyzed the patterns of CNV activity and recurrence in patients treated with ranibizumab over a long period of time and their correlation with their individual VSTs.

\section{Materials and methods}

\section{Study population}

This is a post-hoc analysis of data gathered in a prospective, observational study at the University Hospital of Cologne, Germany. The study was performed in accordance with the tenets of the Declaration of Helsinki. Approval was obtained from the Ethics Committee of the University of Cologne (reference number 11-027), and written informed consent was given by all participants. The study was registered at ClinicalTrials.gov (Identifier NCT01213667).

\section{Inclusion and exclusion criteria}

The study consists of patients 60 years of age or older with active subfoveal CNV secondary to AMD, which was diagnosed by fluorescein angiography (FA) with indocyanine green and spectral-domain optical coherence tomography (SD-OCT). The study eye had to have a bestcorrected visual acuity > 20 Early Treatment of Diabetic Retinopathy Study letters and no previous treatment for nAMD. The minimum follow-up period was 365 days. Exclusion criteria included any previous ophthalmic surgery, except for cataract removal.

\section{Diagnostics and treatment}

Patients initially received three consecutive, monthly intravitreal injections of $0.5 \mathrm{mg}$ ranibizumab. After this first series of treatments, patients were monitored monthly by SD-OCT and fundus examination. FA was repeated in unclear cases. Active $\mathrm{CNV}$ was defined as fluid seen by SD-OCT or leakage seen on FA, or new macular intraretinal or subretinal hemorrhage. Recurrent or persistent activity was again treated with a series of three consecutive, monthly ranibizumab injections.

\section{VEGF suppression time}

The study to determine individual VSTs was published previously. ${ }^{9}$ In brief, aqueous humor was collected immediately before dosing with ranibizumab, and VEGF was quantified with a Luminex xMAP assay (Luminex Corporation, Austin, TX, USA). Repeated measurements of consecutive injections in the same patient at different intervals allowed to determine individual VSTs.

\section{Statistics}

All statistical evaluation was performed at a significance level of a 0.05. Statistical analysis was conducted using SPSS software (v. 21.0; IBM, Chicago, USA).

\section{Results}

In this post-hoc analysis, 73 eyes of 73 patients were included. Ten patients from the original cohort ${ }^{10}$ were excluded because of a follow-up time of $<1$ year due to drop-out from the study. The patients' characteristics are summarized in Table 1.

During the follow-up time, 61 of 73 patients (84\%) demonstrated at least one inactive, dry status in SD-OCT. Twelve patients $(16 \%)$ showed persistent activity. The mean dry interval was 76.5 days from injection to detection of recurrent activity $(S D \pm 136.5$ days, range: 0-829 days). Figure 1 displays the individual range in days of dry intervals until recurrence of CNV activity analyzed by OCT imaging. Although the majority of recurrent activity was found between 2 and 4 months, there were also some cases with a recurrence after a very long dry period of up to 16 months.

The individual range of the length of dry intervals is grouped in Table 2. Overall, $42 \%$ of patients had a range of more than 90 days meaning that $\mathrm{CNV}$ activity was highly variable. Patients with permanently persisting CNV activity or a permanently dry status after the initial treatment were assigned to a value of 0 days.

Table 1 Study population characteristics

\begin{tabular}{llll}
\hline Study participants $(\mathrm{n}=73)$ & & $S D$ & Range \\
\hline Women, $n$ & $41(56 \%)$ & NA & NA \\
Age, mean (years) & 76.8 & \pm 6.8 & $60-95$ \\
Right eyes, $n$ & $39(53 \%)$ & NA & NA \\
Follow-up time, mean (days) & 716.6 & \pm 159.1 & $412-1239$ \\
Number of injections per patient, & 12.9 & \pm 6.3 & $3-26$ \\
mean & & & \\
VST, mean (days) & 36.6 & \pm 7.0 & $26-69$ \\
\hline
\end{tabular}

Abbreviations: VST, vascular endothelial growth factor suppression time; $\mathrm{SD}$, standard deviation. 


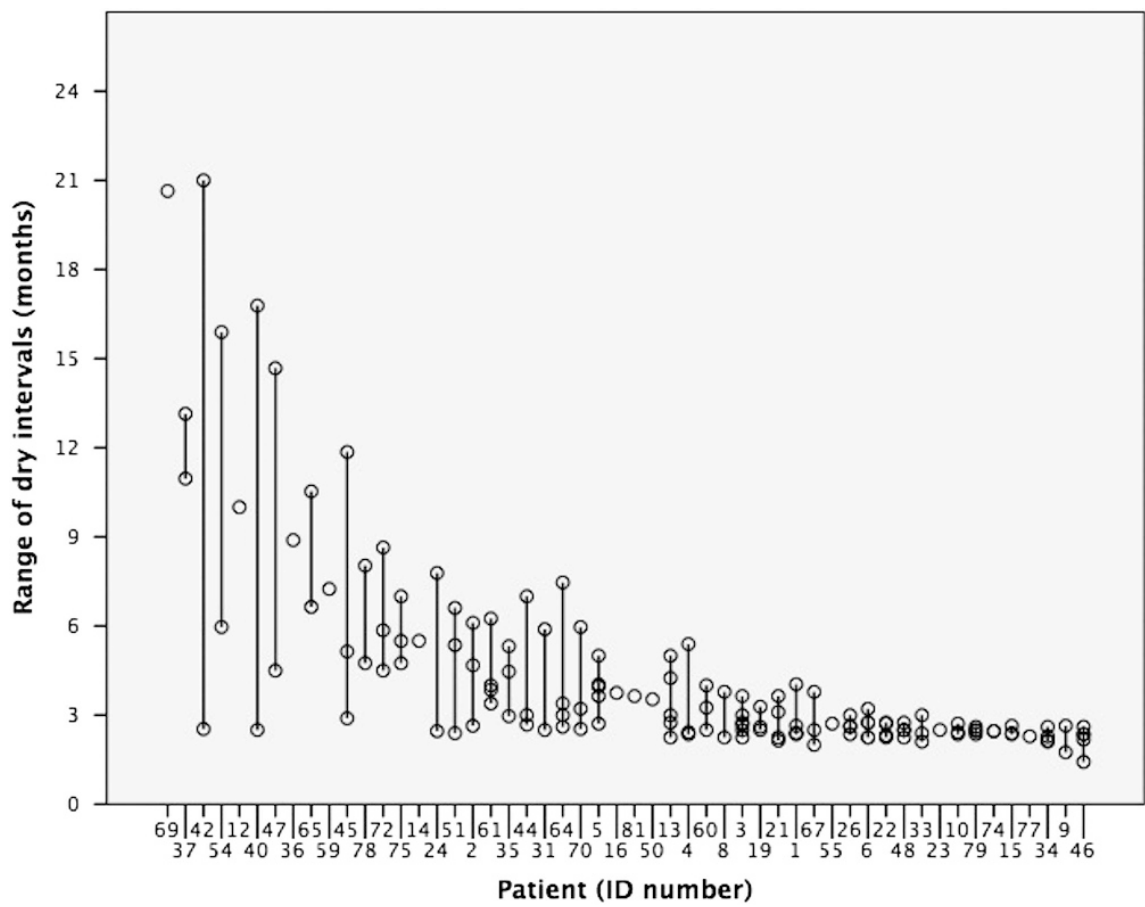

Figure 1 Individual range of length of dry intervals from injection to detection of recurrent activity. Graphs indicate for every individual patient the timing of recurrence (circles) as well as the range of dry intervals in days. Data is sorted in descending order starting longest mean dry interval. Patients with persistent activity and those without recurrence of CNV after initial treatment are not displayed.

Table 2 Intra-individual variability of dry intervals. Patients were grouped by their range of the length of dry intervals after ranibizumab injections

\begin{tabular}{lc}
\hline Individual range of length of dry intervals & $\mathrm{n}$ \\
\hline 0 days (persistent activity) & $12(16 \%)$ \\
0 days (permanently dry after initial three injections) & $9(12 \%)$ \\
$\leq 30$ days & $2(3 \%)$ \\
$31-60$ days & $2(3 \%)$ \\
$61-90$ days & $17(23 \%)$ \\
$91-120$ days & $11(15 \%)$ \\
$>120$ days & $20(27 \%)$ \\
\hline
\end{tabular}

Response patterns to ranibizumab therapy were analyzed individually. The first group were patients that always responded with a dry, inactive status 1 month after every series of three injections $(n=26,36 \%)$. The second group consisted of patients that sometimes reacted with a dry interval but sometimes also showed persistent activity ( $n=35,48 \%)$. The third group included patients with persistent activity with no dry intervals during the whole follow-up period ( $n=12,16 \%$ ).

In a consecutive analysis individual VSTs were correlated with the mean dry interval following ranibizumab treatment but this correlation did not reach significance ( $P=0.12$, Pearson) (Figure 2). Although this pattern analysis shows a correlation of VST and mean dry interval close to the average of both parameters, some individuals present a combination of short VST with long mean CNV-free interval and vice versa.

\section{Discussion}

Regular disease activity would facilitate the treatment of nAMD with anti-VEGF drugs. If recurrences would follow a timely pattern, visits for injections and monitoring could be individually scheduled. We analyzed 73 eyes of 73 patients with monthly SD-OCT examinations, and a follow-up period between 1 and $31 / 2$ years that were treated with ranibizumab.

In our study, CNV activity of nAMD patients showed a high variability. This was shown in irregular $\mathrm{CNV}$ activity with variable dry intervals after ranibizumab injections: $43 \%$ had a range of the duration of these intervals of more than 90 days and $66 \%$ of more than 60 days. So the majority of patients could not be allocated to scheduled examinations longer than 4 weeks in order not to miss a recurrent activity.

Variability became also apparent in the more immediate response to therapy. Although $2 / 3$ of patients 


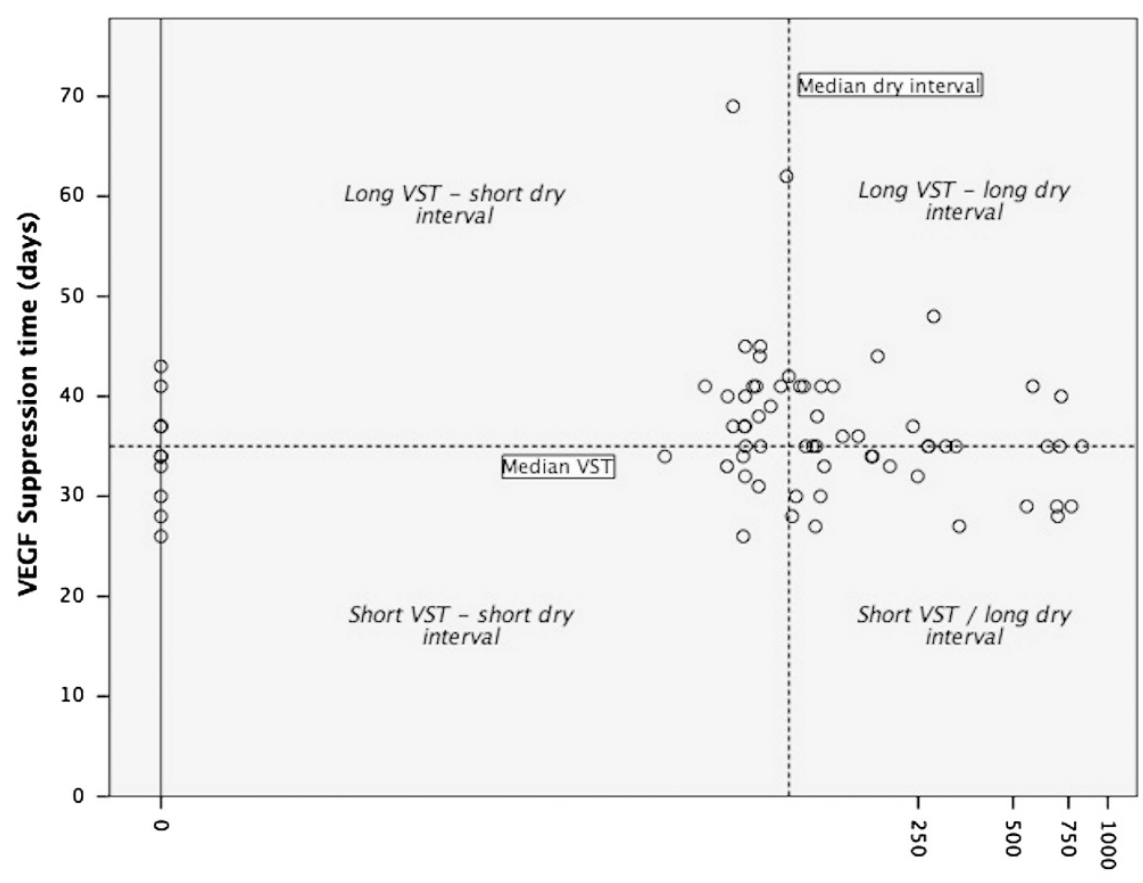

Mean dry interval (days)

Figure 2 Relation between the individual mean free interval until CNV-recurrence in days and the patient-specific intraocular VEGF suppression time (VST) in days.

showed either persistent activity or always were dry after every series of injections, $1 / 3$ of patients responded in a variable fashion with either inactivity or persistence after different series of injections.

These results contrast other studies postulating regular CNV recurrence. ${ }^{11}$ One explanation for the differences could be the longer observation time in our study. Recurrence patterns may only look regular for a short period of time. A limitation of our study was the fixed monthly monitoring visits, which might miss out on some recurrence patterns. On the other hand, as the individual variability in recurrences was so high, this hardly affected the results.

The predictability of the individual CNV activity in nAMD therefore remains challenging. With regard to the high variability of CNV recurrence, close monitoring seems to be necessary as a delay in therapy can lead to irreversible deterioration of vision. ${ }^{2}$

Our study also revealed that even after a long dry period of several months late recurrences can occur. On the basis of this data, it is difficult to recommend a prolongation of monitoring visits or an exit strategy to terminate visits in general. Although very late recurrences are rare and will not affect the visual outcome of a clinical study, they could be very relevant for the individual patient. It also stresses the importance of an individualized approach taking into account patients' preferences.

In general VEGF suppression and VSTs correlate with CNV activity. The correlation of VEGF levels in different compartment of the eye as aqueous humor, vitreous and subRPE is assumed but still not yet fully clear. At last vitreous and anterior chamber show a positive correlation. ${ }^{12-15}$

However, end of VEGF suppression does not mean the immediate appearance of signs of recurrent CNV activity. The presence of VEGF is necessary but not sufficient for recurrences as other growth factors as well as the complement system have an important role in disease activity. As well, individual patients may show persistent CNV activity despite ongoing VEGF suppression. As the mean VST was 37 days and the mean dry interval was 77 days, it becomes understandable that we found no correlation between these two parameters in our study. Furthermore, small differences were most probably smoothed over by examination intervals of 4 weeks.

In conclusion, individual CNV activity in nAMD is irregular, which is reflected in the range of the duration of dry intervals and late recurrences. Any OCT-follow-up scheme screening for recurrences needs to reflect this high variability. 


\section{Summary}

What was known before

- Patients with nAMD are treated with drugs targeting VEGF. Disease activity is monitored by SD-OCT.

- Treatment regimens such as 'treat and extend' are used to reduce the number of examinations and treatments. They assume some regularity in disease activity.

What this study adds

- Disease activity in nAMD is irregular. This is reflected in the range of the duration of dry intervals and late recurrences.

- Any therapy regimen needs to consider this high variability in order not to miss recurrences.

\section{Conflict of interest}

The authors declare no conflict of interest.

\section{Acknowledgements}

This study was supported by a grant from the Novartis. The sponsor had no role in design, conduct, or analysis of the study.

\section{References}

1 Solomon SD, Lindsley K, Vedula SS, Krzystolik MG, Hawkins BS. Anti-vascular endothelial growth factor for neovascular age-related macular degeneration. Cochrane Database Syst Rev 2014; 8: CD005139.

2 Muether PS, Hermann MM, Koch K, Fauser S. Delay between medical indication to anti-VEGF treatment in age-related macular degeneration can result in a loss of visual acuity. Graefes Arch Clin Exp Ophthalmol 2011; 249(5): 633-637.

3 Muether PS, Hoerster R, Hermann MM, Kirchhof B, Fauser S. Long-term effects of ranibizumab treatment delay in neovascular age-related macular degeneration. Graefes Arch Clin Exp Ophthalmol 2013; 251(2): 453-458.

4 Kiss S, Liu Y, Brown J, Holekamp NM, Almony A, Campbell J et al. Clinical monitoring of patients with age-related macular degeneration treated with intravitreal bevacizumab or ranibizumab. Ophthalmic Surg Lasers Imaging Retina 2014; 45(4): 285-291.
5 Ziemssen F, Eter N, Fauser S, Bopp S, Radermacher M, Hasanbasic $\mathrm{Z}$ et al. Retrospective investigation of anti-VEGF treatment reality and effectiveness in patients with neovascular age-related macular degeneration (AMD) in Germany: treatment reality of ranibizumab for neovascular AMD in Germany. Ophthalmologe 2015; 112(3): 246-254.

6 Haller JA. Current anti-vascular endothelial growth factor dosing regimens: benefits and burden. Ophthalmology 2013; 120(5 Suppl): S3-S7.

7 Rayess N, Houston SK 3rd, Gupta OP, Ho AC, Regillo CD. Treatment outcomes after 3 years in neovascular age-related macular degeneration using a treat-and-extend regimen. Am J Ophthalmol 2015; 159(1): 3-8 e1.

8 Berg K, Pedersen TR, Sandvik L, Bragadottir R. Comparison of ranibizumab and bevacizumab for neovascular agerelated macular degeneration according to LUCAS treatand-extend protocol. Ophthalmology 2015; 122(1): 146-152.

9 Muether PS, Hermann MM, Viebahn U, Kirchhof B, Fauser S. Vascular endothelial growth factor in patients with exudative age-related macular degeneration treated with ranibizumab. Ophthalmology 2012; 119(10): 2082-2086.

10 Muether PS, Hermann MM, Droge K, Kirchhof B, Fauser S. Long-term stability of vascular endothelial growth factor suppression time under ranibizumab treatment in agerelated macular degeneration. Am J Ophthalmol 2013; 156(5): 989-993 e982.

11 Mantel I, Deli A, Iglesias K, Ambresin A. Prospective study evaluating the predictability of need for retreatment with intravitreal ranibizumab for age-related macular degeneration. Graefes Arch Clin Exp Ophthalmol 2013; 251(3): 697-704.

12 Aiello LP, Avery R, Arrigg P, Keyt B, Jampel H, Shah S et al. Vascular endothelial growth factor in ocular fluid of patients with diabetic retinopathy and other retinal disorders. $N$ Engl J Med 1994; 331: 1480-1487.

13 Funatsu H, Yamashita H, Noma H, Mimura T, Nakamura S, Sakata $\mathrm{K}$ et al. Aqueous humor levels of cytokines are related to vitreous levels and progression of diabetic retinopathy in diabetic patients. Graefes Arch Clin Exp Ophthalmol 2005; 243: 3-8.

14 Noma H, Funatsu H, Mimura T, Harino S, Hori S. Aqueous humor levels of vasoactive molecules correlate with vitreous levels and macular edema in central vein occlusion. Eur J Ophthalmol 2010; 20: 402-409.

15 Ecker SM, Hines JC, Pfahler SM, Glaser BM. Aqueous cytokine and growth factor levels do not reliably reflect those levels found in the vitreous. Mol Vis 2011; 17: 2856-2863. 